\title{
Computer Based Performance Evaluation of Segmentation Methods for Chest X-Ray Image
}

\author{
Manoj R. Tarambale and Nitin S. Lingayat
}

\begin{abstract}
According to WHO (World Health Organization) report, because of chest diseases more than 12 million death cases are reported during the year 2008 . If chest disease is detected in its early stage then the possibility of surviving the patient is more. One of the most preferred scan for the early detection of chest disease is $\mathrm{X}$-ray scan. $\mathrm{X}$-ray is inexpensive, painless and required less time to generate image. $X$-ray image contain a lot of irrelevant information and has intensity problems, which makes the task of locating and analyzing suspicious area difficult by the doctor. By using pre-processing and segmentation technique, suspicious area is easily separate out from the rest of the structure. In the present paper, segmentation techniques and the segmentation results after applying on the X-ray images are discussed. In segmentation, image is partition into a meaningful region. The result of image segmentation is a set of segments that collectively cover the entire image and all pixel in the segmented region which are similar with respect to some characteristic or computed property such as color, intensity, texture etc. In present paper, some of the segmentation techniques such as edge detection, thresholding, skeletonization, contour and watershed transform are applied on the chest X-ray image and the effectiveness of each techniques are shown with the help of images and properties extracted.
\end{abstract}

Index Terms-Anatomical, contour, segmentation, skeletonization, thresholding.

\section{INTRODUCTION}

$\mathrm{X}$-ray is an electromagnetic radiation, which differentially penetrates structures within the body and creates images of these structures on photographic film or a fluorescent screen. Diagnostic X-rays are useful in detecting abnormalities within the body. They are painless and non-invasive way to help diagnose problems such as presence of foreign bodies, abnormalities, fractures etc. X-ray scans are very popular worldwide because of simple operation and scanning centers are easily available in / near rural and urban areas. X-ray techniques is less expensive and required less time to generate images as compared to other scans (CTs, MRIs and PETs) but on the other hand, X-ray images are very sensitive to noise. X-ray image diagnosis become difficult, if it contains lot of irrelevant information. Some time high or low contrast and brightness makes the task of detecting suspicious area difficult. Success of any bio-medical image

Manuscript received March 23, 2013; revised June 20, 2013

Manoj R. Tarambale is with Marathwada Mitra Mandal's college of Engineering, Pune, Pin-411052, University of Pune, India (e-mail: manoj_tarambale@yahoo.com).

Nitin S. Lingayat is with Electrical Department, DR. Babasaheb Ambedkar Technological University's Institute of Petrochemical Engineering, Lonere, Pin-402103 India (e-mail: nslingayat@yahoo.com). processing system depends upon the segmentation step. If segmentation is done properly then only the further analysis will give correct results. Before doing segmentation, pre-processing (Filtering and contrast stretching) is done to remove irrelevant information and enhance the relevant information.

Segmentation is the process of partitioning a digital image into multiple segments i.e. sets of pixels (also known as super pixels) [1]. The goal of segmentation is to simplify and / or change the representation of an image into something that is more meaningful and easier to analyze [2]. Image segmentation is typically used to locate objects and boundaries (lines, curves etc.) in images. More precisely, image segmentation is the process of assigning a label to every pixel in an image such that pixels with the same label share certain visual characteristics. This is typically used to identify objects or other relevant information in digital images. The result of image segmentation is a set of contours extracted from the image.

The role of segmentation in diagnosing the chest X-ray image is to subdivide the objects in an image. In case of medical image segmentation the aim is to [3]:

1) Study anatomical structures.

2) Identify Region of Interest i.e. locate tumor, lesion and other abnormalities.

3) Measure tissue volume to measure growth of tumor (also decrease in size of tumor with treatment).

4) Help in treatment planning prior to radiation therapy, in radiation dose calculation.

5) Helps to distinguish one anatomic structure from other.

The paper is organized as follows - Section II describes the edge detection techniques as well as the advantage and disadvantages of all the describe edge detection techniques. Section III describes the skeletonization technique. Section IV describes the thresholding and Section V describes the watershed transform. Section VI and Section VII include contour and boundary detection techniques. Section VIII and IX include result and discussion and conclusion.

\section{Edge Detection}

Stepped edges, lines and junction usually convey the most relevant information of an image; hence it is important to detect them in a reliable way [4], [5]. Edge detection has been extensively analyzed in computer vision. The success of higher level processing yields good edges. Edge is defined as an image point where gradient of image intensity function reaches to its local maximum value. Edges are the curves where rapid changes occur in brightness or in the spatial derivatives of brightness [6]. The changes in the brightness are the place in the image is because of: 
1) Surface orientation changes discontinuously

2) One object occludes another

3) A cast shadow line appears

4) There is a discontinuity in surface reflectance properties.

For segmenting the image, computer vision locates a discontinuity in image brightness or its derivatives. Edge detection is the technique that yields pixels lying only on the boundary between regions. In practice, this set of pixels seldom characterizes a boundary completely because of noise, breaks in the boundary from non-uniform illumination and other effects that introduce spurious intensity discontinuities.

Various early edge detectors present distinct and different responses to the same image, showing different details [6], [7]. The edge detection methods can be classified into two types, directional operators and non-directional operators. A directional operator looks for local zero-crossings. In this process, two masks and two convolutions are used. Non-directional or gradient-based operator uses single mask and convolution, but they are sensitive to noise due to gradient nature of the operators [8], [9]. Several edge detectors have been proposed in the literature [10]. The popular gradient operators are that of Sobel, Prewitt, Robert, Laplacian etc. The operator based on derivatives of Gaussian is Laplacian of Gaussian [10].

All edge detectors utilize thresholding of the image for edge detection. Each pixel in the image is compared with this threshold value. If the pixel's intensity is higher than the threshold value then the pixel is set to white in the output image. If it is less than the threshold then it is set to black. The efficient selection of single threshold value is the most important and the most difficult process in edge detection technique. Edge detector based on local technique uses local feature for selecting threshold value. Similarly, edge detector based on global technique uses global feature for selecting threshold value. The most popular methods are based on gradients of the image which are sensitive to changes in the intensity values of the image. The original image is filtered with a suitable mask in the gradient operators. Edge points are detected in places where the original image has a high rate of intensity change. Edge points are detected by selecting an optimum global threshold value. Images contain variations at different levels. Use of a single global threshold over the whole image gives poor results. To avoid this locally derived threshold based on the local mean value is used [11]. But local threshold method also detects false edges due to noise. To avoid this, a global threshold based on variance filtering is used [12]. Popular edge detection technique is discussed below.

\section{A. Robert Edge Detection}

The main objective is to determine the difference between adjacent pixels, one way to find an edge is to explicitly use $\{+1,-1\}$ that calculates the difference between adjacent pixels. In practice, the Robert kernel is too small to reliably find edges in the presence of noise [13], [14]. By using the Robert cross gradient operator, the first order partial derivative is

$$
\frac{\partial f}{\partial x}=f(i, j)-f(i+1, j+1)
$$

and

$$
\frac{\partial f}{\partial x}=f(i+1, j)-f(i, j+1)
$$

The partial derivatives given above can be implemented by approximating them to $2 \times 2$ masks. The Roberts operator masks are given by

$$
G_{x}=\left[\begin{array}{cc}
-1 & 0 \\
0 & 1
\end{array}\right] \quad \text { and } \quad G_{y}=\left[\begin{array}{cc}
0 & -1 \\
1 & 0
\end{array}\right]
$$

\section{B. Sobel Edge Detector}

The Sobel edge detection based on Sobel operator has advantages of emphasizing the central part of the edge. It relies on central differences but gives greater weight to the central pixels when averaging [13], [14]. The partial derivatives of the Sobel operator are calculated as

$$
G_{x}=\left(a_{2}+2 a_{3}+a_{4}\right)-\left(a_{0}+2 a_{7}+a_{6}\right)
$$

and

$$
G_{y}=\left(a_{6}+2 a_{5}+a_{4}\right)-\left(a_{0}+2 a_{1}+a_{2}\right)
$$

The Sobel masks in the $3 \times 3$ matrix form are given as

$$
G_{x}=\left[\begin{array}{ccc}
-1 & -2 & -1 \\
0 & 0 & 0 \\
1 & 2 & 1
\end{array}\right] \quad \text { and } \quad G_{y}=\left[\begin{array}{ccc}
-1 & 0 & 1 \\
-2 & 0 & 2 \\
-1 & 0 & 1
\end{array}\right]
$$

\section{Prewitt Edge Detector}

The Prewitt edge detector based on Prewitt operator approximates the first derivatives. This operator does not place any pixels those are closer to the center of the mask. The Prewitt edge detector masks are one of the oldest and best understood methods of detecting edges in image. Basically there are two masks, one for detecting image derivatives in $\mathrm{X}$ direction and other one for detecting image derivatives in $\mathrm{Y}$ direction. The results of Prewitt edge detection are threshold in order to produce a discrete set of edges [13], [14]. The direction of gradient mask is given by the mask giving maximal response. The arrangement of pixels about the central pixel $[i, j]$ as shown below:

$$
\left[\begin{array}{ccc}
a_{0} & a_{1} & a_{2} \\
a_{7} & {[i, j]} & a_{3} \\
a_{6} & a_{5} & a_{4}
\end{array}\right]
$$

The partial derivates of the Prewitt operator are calculates as

$$
G_{x}=\left(a_{2}+c a_{3}+a_{4}\right)-\left(a_{0}+c a_{7}+a_{6}\right)
$$

and

$$
G_{y}=\left(a_{6}+c a_{5}+a_{4}\right)-\left(a_{0}+c a_{1}+a_{2}\right)
$$

The constant ' $c$ ' in the above expression implies the emphasis given to pixels closer to the centre of the mask $G_{x}$ and $G_{y}$ are the approximations at $[i, j]$. 
Setting $c=1$, the Prewitt operator mask is obtained as

$$
G_{x}=\left[\begin{array}{ccc}
-1 & -1 & -1 \\
0 & 0 & 0 \\
1 & 1 & 1
\end{array}\right] \quad \text { and } \quad G_{y}=\left[\begin{array}{ccc}
-1 & 0 & 1 \\
-1 & 0 & 1 \\
-1 & 0 & 1
\end{array}\right]
$$

The Prewitt mask differentiates in one direction and averages in other direction, so edge detection is less vulnerable to noise.

\section{Laplacian of Gaussian Operator ( $L o G)$}

Laplacian operator is a second order derivative operator. It subtracts the brightness values of each of the neighboring pixels from the central pixel. When a discontinuity is present within the neighborhood in the form of a point, line or edges, the result of the Laplacian is a non-zero value. It may be either positive or negative depending whether the central point lies with respect to the edges. It is rotationally invariant. The Laplacian operator does not depend on direction as long as they are orthogonal [15].

Laplacian operator can be expressed in terms of different equation as given below

$$
\begin{gathered}
\frac{\partial f}{\partial x}=f(x+1, y)-f(x, y) \\
\frac{\partial^{2} f}{\partial x^{2}}=f(x+1, y)-2 f(x, y)+f(x-1, y)
\end{gathered}
$$

Similarly

$$
\frac{\partial^{2} f}{\partial y^{2}}=f(x, y+1)-2 f(x, y)+f(x, y-1)
$$

That implies that

$$
\begin{aligned}
\nabla_{f}^{2}= & {[f(x+1, y)+f(x-1, y)+f(x, y+1)+} \\
& f(x, y-1)]-4 f(x, y)
\end{aligned}
$$

The $3 \times 3$ Laplacian operator is given by

$$
\left[\begin{array}{ccc}
-1 & -1 & -1 \\
-1 & 8 & -1 \\
-1 & -1 & -1
\end{array}\right]
$$

Image coordinates under the mask

$$
\left[\begin{array}{ccc}
(x-1, y-1) & (x-1, y) & (x-1, y+1) \\
(x, y-1) & (x, y) & (x, y+1) \\
(x+1, y-1) & (x+1, y) & (x+1, y+1)
\end{array}\right]
$$

A prominent source of performance degradation in the Laplacian operator is noise in the input image. The noise effect can be minimized by smoothing in the image prior to edge enhancement. The Laplacian of Gaussian (LoG) operator smoothes the image through convolution with a Gaussian shaped kernel followed by applying the Laplacian operator.

\section{E. Canny Edge Detection}

Canny edge detector defines edges as zero-crossings of second derivatives in the direction of the greatest first derivative. The Canny edge detector operator works in a multi-stage process [16]. First, the image is smoothed by a Gaussian convolution and then, a 2D first derivative operator is applied to smoothed image to highlight regions of the image with high spatial derivatives. Edges give rise to ridges in the gradient magnitude image. The algorithm then tracks along the top of the ridges and sets to zero all pixels that are not actually on the ridge top so as to give a thin line in the output, a process known as non-maximal suppression. The tracking process exhibits hysteresis controlled by two thresholding $T 1$ and $T 2$ with $T 1>T 2$. Tracking can only begin at a point on a ridge higher than $T 1$. Tracking then continues in both directions out from that point until the height of the ridge falls below $T 2$. This hysteresis helps to ensure that noisy edges are not broken into multiple edge fragments. The effectiveness of a Canny edge detector is determined three parameters-width of the Gaussian kernel upper threshold and lower threshold used by the tracker .

Increasing the width of the Gaussian kernel reduces the detector's sensitivity to noise, at the expense of losing some of the finer details in the image. The localization error in the detected edges also increases slightly as the Gaussian width is increased. The Gaussian smoothing in the Canny edge detector fulfills two purposes. First, it can be used to control the amount of details that appears in the edge image and second it can be used to suppress noise.

The upper tracking thresholding is usually set quite high and lower threshold value is set quite low for good result. Setting the lower threshold too high will cause noisy edges to break up. Setting the upper threshold too low increases the number of spurious and undesirable edge fragments appearing in the output.

\section{SKELETONIZATION}

In many image processing application, it is desired to identify certain objects located in an image. Using every pixel in each object to identify an object increases the complexity of identifying process. An important approach for representing the structural shape of a plane region is to reduce it to graph. Skeletonization of an image is unique geometrical description that requires far pixels than the original image [17].

The skeleton of a region may be defined via the medial axis transform (MAT) [18]. The MAT of a region $\mathrm{R}$ with border $\mathrm{B}$ is as follows. For each point $\mathrm{p}$ in $\mathrm{R}$, the closest neighbor is found in $\mathrm{B}$. If $\mathrm{p}$ has more than one such neighbor, it is said to belong to the medial axis of $\mathrm{R}$.

Although the MAT of a region yields an intuitively pleasing skeleton, direct implementation potentially involves calculating the distance from every interior point to every point on the boundary of a region. The skeletonization for chest medical X-ray image is shown in Fig. $1 \mathrm{~m}$ ).

\section{THRESHOLDING}

Thresholding technique produce segmented area which are 
having pixels with similar intensities. Thresholding is a useful technique for establishing boundaries in images that contain solid objects resting on a contrasting background. There exist a large number of gray-level based segment methods using either global or local image information. The thresholding technique requires an object with homogenous intensity and a background with a different intensity level. Such an object can be segmented into two regions by simple thresholding. Because of its intuitive properties, simplicity of implementation and computational speed image thresholding enjoys a central position in applications of image segmentation.

Suppose that the intensity histogram corresponds to an image $f(x, y)$ composed of light objects on a dark background, in such a way that object and background pixels have intensity values grouped into two domain modes. To extract the objects from the background is to select threshold, $\mathrm{T}$ that separate these modes [19]. Then any point $(x, y)$ in the image at which $f(x, y)>T$ is called an object point otherwise the point is called back-point. Segmented image, $g(x, y)$ given by

$$
g(x, y)=\left[\begin{array}{lll}
1 & \text { if } & f(x, y)>T \\
0 & \text { if } & f(x, y) \leq T
\end{array}\right]
$$

When $T$ is a constant applicable over an image, the process is referred to as global thresholding. When the value of $\mathrm{T}$ changes over an image it is referred as variable thresholding. The term local or regional thresholding is used sometimes to denote variable thresholding in which the value of $T$ at any point $(x, y)$ in an image depends on properties of a neighborhood of $(x, y)$. If $T$ depends on the spatial coordinates $(x, y)$ themselves then the variable thresholding is often referred to as dynamic or adaptive thresholding. Multiple thresholding is given by

$$
g(x, y)=\left[\begin{array}{rr}
a & \text { if } f(x, y)>T_{2} \\
b & \text { if } T_{1}<f(x, y) \leq T_{2} \\
c & \text { if } f(x, y) \leq T_{1}
\end{array}\right]
$$

where $a, b$ and $c$ are any three distinct intensity values and $T 1$, $T 2$ are the two threshold. The thresholding for chest medical $\mathrm{X}$-ray image is shown in Fig. 1 a).

\section{WATERSHED TRANSFORM}

It is a powerful tool for image segmentation. In a watershed transformation, the image is considered as a topographic surface. The region with constant gray level of the image represents the altitude. The region with constant gray level constitutes the flat zone of an image. Also region an edge corresponds to high water sheds and low gradient region interiors corresponds to catchment basins. Catchments basins of the topographic surface are homogeneous in the sense that all pixels belonging to the same catchment basin are connected with the basin of region of minimum altitude by a simple path of pixels that have a monotonically decreasing altitude along the path. Such catchment basins represent the regions of the segmented image. By viewing the intensity in an image as elevation and simulating rainfall, it is possible to decompose an image into watershed region [17].

Watersheds are defined as lines separating catchment basin, which belongs to different minima. A watershed can be imaged as a high mountain that separates two regions. Each region has its own minimum and if a drop of water falls on one side of the watershed, it will reach the minimum of the regions. The regions that the watershed separates are called catchment basins. The watershed result for chest medical $\mathrm{X}$-ray image is shown in Fig. $1 \mathrm{c})-1 \mathrm{e}$ ).

\section{CONTOUR}

Contour is a two-dimensional plot which shows the one-dimensional curves on which the plotted quantity ' $q$ ' is a constant [17]. These curves are defined by

$$
q(x, y)=q_{j}, j=1,2, \ldots, N c
$$

where ' $N c$ ' is the number of contours that are plotted. These curves of constant ' $q$ ' are known as the "contours" of $q$ or as the "isolines" of ' $q$ ' or as the "level surfaces" of ' $q$ '. The contour plot for chest medical X-ray image is shown in Fig. 1 n).

\section{BOUNDARY}

Boundary detection is a morphological operation and is very effective in detecting boundaries in a binary image $X$ [20]. Following algorithm is widely used in detecting boundary

$$
\begin{aligned}
& Y=X-(X \Theta B) \\
& Y=(X \oplus B)-X
\end{aligned}
$$

or

$$
Y=(X \oplus B)-(X \Theta B)
$$

where $Y$ is the boundary image, operator ' $\Theta$ ' denotes erosion and operator ' $\oplus$ ' denotes dilation. '-' denotes the set theoretical subtraction.

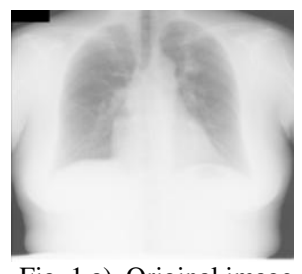

Fig. 1 a). Original image.

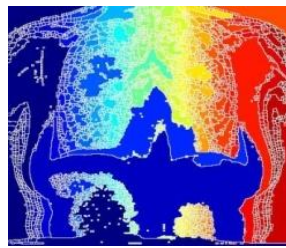

Fig. 1 c). Watershed gradient Calculated rom the $f$ Original image

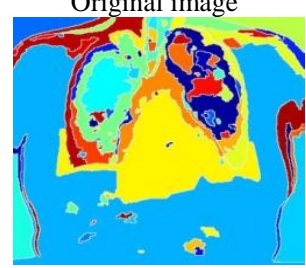

Fig. 1 e). Color impose on watershed image.

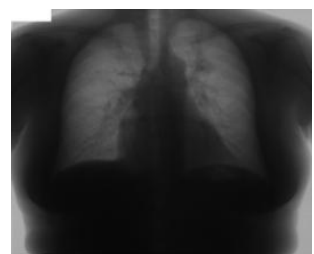

Fig. 1 b). Negativity of original image.

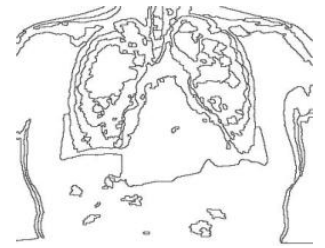

Fig. 1 d). Original image after applying watershed algorithm

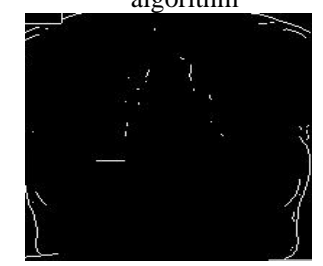

Fig. $1 \mathrm{f})$. Sobel edge detection 


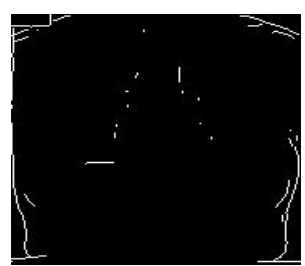

Fig. $1 \mathrm{~g})$. Prewitt edge detection

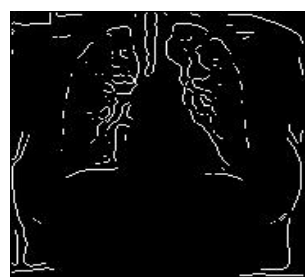

Fig. 1 i). LoG edge detection.

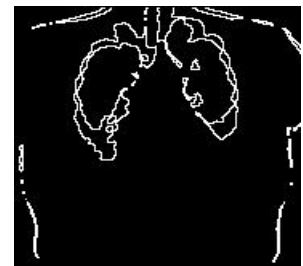

Fig. 1 k). Boundary detection.

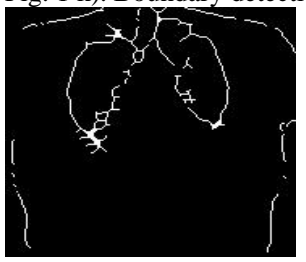

Fig. $1 \mathrm{~m})$. Skeletonization.

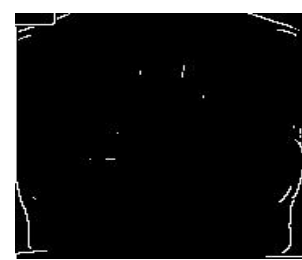

Fig. 1 h). Robert edge detection.

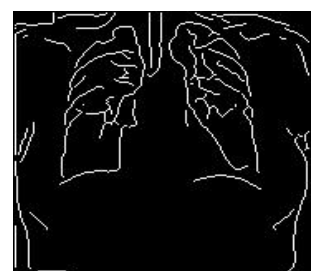

Fig. 1 j). Canny edge detection.

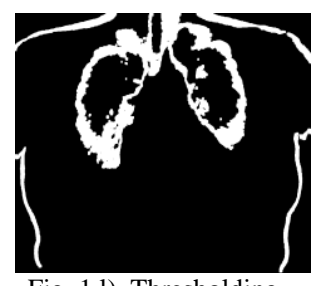

Fig. 1 1). Thresholding.

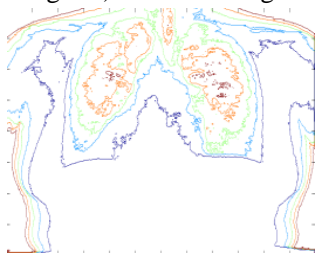

Fig. 1 n). Contour plot.

\section{COMPARISON OF EdGE DETECTION TECNIQUe}

Table I shows the calculated parameter from the edge detected images [21]. These calculated parameters are used for comparing the edge detection technique.

\section{RESUlT AND DisCUSSION}

$\mathrm{X}$-ray imaging is the fastest and inexpensive technique for early detection of suspicious area of disease. Easily available scanning centre and less cost of X-ray imaging makes it popular among the poor people's of developing country. For analyzing suspicious area from the chest X-ray image, segmentation is done. Lot of irrelevant information is present in X-ray image, which makes the process of diagnosis difficult. Segmentation is used to localize suspicious area from the image and also helps in separating the suspicious area from the remaining structure. In the present paper, edge detection methods, skeletonization, contour, thresholding and boundary detection are some of the method for segmentation were discussed [22]. Results of all segmentation techniques are shown with the help of images Fig. 1 a)-Fig. 1 n). Table I, shows various parameters comparative values for edge detection.

One of the biggest problems with the edge detection technique is that they are very sensitive to noise because of this, sometime edge detector operator detect false edges. Similarly, when there is a little change in the intensity between two objects, some edge detectors may fail in detecting this small difference as an edge of the object.

Sobel, Prewitt and Robert are very sensitive to noise and they can detect edges in a specific direction. These operators detect only strong edges. But on the other hand implementation of this operator is simple and less complex. Robert edge operators have the smallest mask order, thus the position of the edges is more accurate, but the problem with the short of the operator is its vulnerability to noise. Prewitt mask is less vulnerable to noise than Robert. The Sobel operator is based on convolution in a very small neighborhood and work well for specific image only. The biggest disadvantages of this edge detection are its dependence on the size of object and sensitivity to noise. It yields the best result when image does not contain noise.

TABLE I: COMPARISON OF EDGE DETECTION TECHNIQUE

\begin{tabular}{|c|c|c|c|c|c|c|}
\hline & $\begin{array}{l}\text { No of } \\
\text { edges }\end{array}$ & $\begin{array}{c}\text { Sum of the area } \\
\text { of all edges }\end{array}$ & $\begin{array}{c}\text { Sum of the perimeter } \\
\text { of all edges }\end{array}$ & $\begin{array}{l}\text { Edge detected } \\
\text { of max. area }\end{array}$ & $\begin{array}{c}\text { Edge detected } \\
\text { of max. } \\
\text { perimeter }\end{array}$ & Entropy \\
\hline Sobel & 167 & 2136 & 4246.9 & 460 & 943.2376 & 0.0710 \\
\hline Prewitt & 142 & 2097 & 4204.4 & 460 & 943.2376 & 0.0699 \\
\hline Robert & 104 & 1620 & 2863.1 & 405 & 805.6569 & 0.0564 \\
\hline LoG & 1160 & 11648 & 2338.7 & 358 & 719.7990 & 0.2717 \\
\hline Canny & 337 & 9567 & 2097.7 & 543 & 1115.5 & 0.2343 \\
\hline
\end{tabular}

Drawback of the Laplacian operator is that it does not give useful directional information. The Laplacian, being an approximation to the second derivative, it doubly enhances noise in the image. By using Gaussian filter noise is removed from the image. LoG (Laplacian of Gaussian) operator is malfunctioning at the corners, curves and where the gray level intensity function varies. Not able to find the orientation of edge because of using the Laplacian filter. In LoG detector, all possible edges are detected. It is more effective in detecting edges than Sobel, Prewitt and Robert operators.
Canny operator detects edges in all possible direction but the implementation is very complex and very difficult [23], [24].

Thresholding technique is simple to implement but it is difficult to decide exact threshold value. If the exact value of thresholding is not determined then the image may be over threshold or below threshold. In thresholding, image can be divided into two or three gray level pixel. If image contain random pixel values, in such case thresholding cannot be used for segmenting particular region from the image.

The drawbacks of watershed algorithm based 
segmentation are 1) over-segmentation 2) sensitivity to noise 3 ) poor performance in the area of low contrast boundaries 4) poor detection of thin structures. The basins watershed algorithm works well if each local minima corresponds to an object of interest. In such cases the watershed lines represent the boundaries of the objects. If there are many more minima in the image than the object of interest, the image will be over segmented. The central problem of the contour plot is that if the contour lines are not somehow labeled with additional text, color or line style, it is not very informative.

Skeletonization provides a simple and compact representation of a shape that preserves many of the topological and size characteristics of the original shape. From skeletonization, a rough idea is generated about the length of a shape by considering just the end points of the skeleton and finding the maximally separated pair of end points on the skeleton. In Boundary technique only the boundary is preserve and rest of the pixel other than boundary is set to zero gray value pixel. It only provides boundary information. If image contain lot of noise or very small structure then the extraction of the boundary is difficult and even if the boundary is extracted then it will not provide any relevant information.

Above discuss techniques give a rough idea about the shape, size and location of the suspicious area [24]. Result of the above technique is used for calculating features, which further helps in classifying the suspicious area and gives a conformation about the disease.

All above techniques are implemented with the help of MATLAB software using Digital Image Processing toolbox.

\section{CONCLUSION}

This paper describes the various image segmentation techniques. Discuss above techniques, are applied to chest $\mathrm{X}$-ray image and results are shown with the help of images. All techniques are successfully helps in segmenting image. If image is not noise free and the images contain random pixel values then there is a possibility of finding false positive or false negative structure. It helps in isolating even a small region from the entire image.

\section{ACKNOWLEDGMENT}

We would like to express our deepest appreciation to the Japanese Society of Radiological Technology (JSRT) in cooperation with the Japanese Radiological Society (JRS) for providing clinically well proven images for research purpose.

\section{REFERENCE}

[1] P. Thakare, "A study of image segmentation and edge detection techniques," International Journal on Computer Science and Engineering (IJCSE), vol. 3, no. 2, pp. 899-904, Feb. 2011.

[2] G. A, Kudale and M. D. Pawar, "Study and analysis of various edge detection methods for X-ray images," International Journal of Computer Science and Application, pp. 15-19, 2010.

[3] N. Sharma and L. M. Aggarwal, "Automated medical image segmentation techniques," Journal of Medical Physics, vol. 35, no.1, pp. 3-14, Mar. 2010.

[4] N. Mann and P. Singh, "Medial axis transformation based skeletonization of image patterns using image processing techniques," International Journal of Science and Research (IJSR), vol. 1, issue 3, pp. 220-223, Dec. 2012.
[5] D. Burr and M. C. Morrone, "A nonlinear model of feature detection," Nonlinear Vision, Determination of Receptive Field, Function and Networks, Boca Raton, FL: CRC, 1992.

[6] M. Brejil, "Comprehensive Exam," Ph.D. dissertation, Dept. of Electrical and Computer Engineering University of Iowa, March 9, 1998.

[7] M. A. Sid-Ahmed, "Performance comparison of k-means \& canny edge detection algorithm on MRI images", International Journal of Mobile Network Communications \& Telematics (IJMNCT), McGraw-Hill, Inc Anu Sharma and Gulista, vol. 1, no. 1, pp. 1-12, Sep. 2011.

[8] T. Zuva, O. O. Olugbara, S. O. Ojo, and S. M. Ngwira, "Image segmentation, available techniques, developments and open issues," Canadian Journal on Image Processing and Computer Vision, vol. 2, no. 3, pp. 20-29, March 2011.

[9] R. C. Gonzalez and R. E. Woods, Digital Image Processing, $3^{\text {rd }}$ ed., Delhi, India: Pearson Education, 2009, ch. 10, pp. 711-783.

[10] M. B. Ahmad and T.-S. Choi, "Local threshold and boolean function based edge detection," IEEE Transactions on Consumer Electronics, vol. 45, no. 1, pp. 674-679, Aug. 1999.

[11] A. Elmabrouk and A. aggoun, "Edge detection using local histrogram analysis," Electronics Letters, vol. 34, no. 12, pp. 1216-1217, June 1998.

[12] V. Torre and T. A. Poggio, "On edge detection," IEEE Trans. On Pattern Anal. Machine Intell, vol. 8, issue 2, pp. 147-163, Mar. 1986.

[13] S. D. Olabarriagaa and A. W. M. Smeulders, "Interaction in the segmentation of medical images: A survey," Medical Image AnalysisElsevier Science, vol. 5, no. 1, pp.127-142, July 2001

[14] B. Therese and S. Sundaravadivelu, "Bipolar incoherent image processing for edge detection of medical images," International Journal of Recent Trends in Engineering, vol. 2, no. 2, pp. 229-232, Nov.2009.

[15] S. Jayaraman and T. Veerakumar, Digital Image Processing, $3^{\text {rd }}$ ed., New Delhi, India: Tata McGraw Hill, 2010, ch. 5, pp. 243-273

[16] G. T. Shrivakshan and C. Chandrasekar, "A comparison of various edge detection techniques used in image processing," International Journal of Computer Science Issues(IJCSI), vol. 9, issue 5, no. 1, pp. 269-276, Sep. 2012.

[17] R. C. Gonzalez and R. E. Woods Digital Image Processing Using Matlab, $5^{\text {th }}$ ed., Delhi, India: Pearson Education, 2009, ch. 11, pp. 535-575

[18] B. Vanajakshi and B. Sujatha, "An analysis of thinning and skeletonization for shape representation," International Journal of Computer Communication and Information System (IJCCIS), vol. 2, no. 1, pp. 250-253, Dec. 2010.

[19] S. S. Al-amri, N. V. Kalyankar, and S. D. Khamitkar, "Image segmentation by using thershod techniques," Journal of Computing, vol. 2, issue 5, pp. 83-86, May 2010.

[20] Y.-Q. Zhao et al., "Medical images edge detection based on mathematical morphology," presented at $27^{\text {th }}$ Annual IEEE Engineering in Medicine and Biology Conf., Shanghai, China, Sep. 1-4, 2005 .

[21] M. Juneja and P. S. Sandhu, "Performance evaluation of edge detection techniques for images in spatial domain," International Journal of Computer Theory and Engineering, vol. 1, no. 5, pp. 614-621, Dec. 2009.

[22] S. K. Singh and A. Kathane, "Various methods for edge detection in digital image processing," IJCST, vol. 2, issue. 2, pp. 188-190, June 2011.

[23] S. Kamadi and R. K. Kroshna, "Image segmentation and region growing algorithm," International Journal of Computer technology and Electronics Engineering (IJCTEE), vol. 2, issue 1, pp. 103-107, Oct. 2011.

[24] F. A. Pellegrino, W. Vanzella, and V. Torre, "Edge detection revisited," IEEE Transactions on Systems, Man, and Cybernetics, vol. 34, no. 3, pp. 1500-1518, June 2004

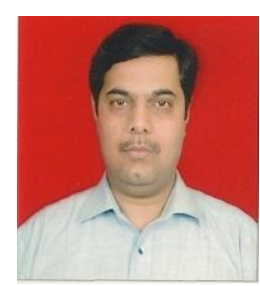

Manoj R. Tarambale was born in Jalgaon District, India, in 1970. He received graduate degree (B.E.) in electrical engineering from BVCOE, Pune-43, University of Pune, India, in 1992 and post graduate degree (M.E.) in control system from WCOE, Sangli, Shivaji University, Kolhapur, India in 2002. Currently, he is pursuing his Ph.D. degree from PACIFIC University, Udaipur, India.

He has one year industrial experience and twenty years teaching experience. At present, he is Assistant Professor and Head of electrical engineering department of Marathwada Mitra Mandal's College of Engineering, Pune-52, University of Pune, India. His main research interests 
are bio-medical image processing, electronic instrumentation, process control instrumentation and medical diagnosis.

Prof. Tarambale is a member of Institute of Engineers (India) and Indian Society for Technical Education (ISTE).

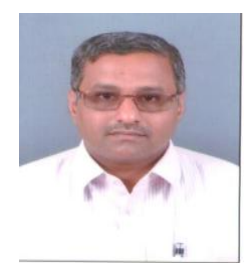

Nitin S. Lingayat was born in Shahapur (Thane), India in 1971. He received the B.E. degree in electrical engineering from the University of Poona, Pune in 1992 and the M.Tech degree from Indian Institute of Technology Bombay, Mumbai in 1998. $\mathrm{He}$ obtained $\mathrm{Ph} . \mathrm{D}$. degree in electrical engineering from J.N. Vyas University, Jodhpur, India in 2008. $\mathrm{He}$ is a head in Electrical Engineering Department, Institute of Petrochemical Engineering of Dr. Babasaheb Ambedkar Technological University, Lonere, Maharashtra (India) from January 2004
He has a teaching experience of twenty one years. His research interest includes biomedical signal and image processing, energy management, statistical signal processing, non-conventional energy sources etc.

Dr. Lingayat is a member of IAENG, Institute of Engineers (India), Indian Society for Technical Education (ISTE). He has published many papers in the National and International Journals. 